\title{
Digital campus data grid environment architecture based on IPV6
}

\author{
RongYi He
}

\author{
Department of Network \& Information Center, Shan Dong University at WeiHai, WeiHai, China \\ hry@sdu.edu.cn
}

Key words: CN (Campus Network); IPv4; IPv6; Internet

\begin{abstract}
Next-generation internet shall completely change the lifestyle of people. IPv6 is very suitable for the large-scale network environment with abundant terminal data on account of the huge address space owned by IPv6, plug and play and easy configuration, and internal support on mobility. With the popularity of IPv6 terminal, people shall enter to a new era with ubiquitous internet and intelligence. This time, the study of my graduation thesis is mainly for the construction of IPv6 campus network in domestic colleges, which can make CERNET2 IPv6 access router to achieve the connection of IPv6, providing the preparations for the large-scale deployment of IPv6 in school by carrying out the researches, education and application developments related to IPv6.
\end{abstract}

\section{Introduction}

At present, the IP address that internet can contain was developed in the 1980s on the basis of Internet Protocol version 4 (IPv4), which adopts the address length of 32-bit about 4.3 billion IP addresses, of which about $70 \%$ are owned by USA who develops such technology, however, our country only have more than 30 million. IPv6 adopts the address length of 128-bit with 64 billion IP addresses (shall be about $3.4 \times 1038$ ), which has greatly improved the whole handing capacity of internet and preferably ensured the security at the same time. For any on-line computer or telephone, an IP address shall be obtained as a link point on vast internet. Some people even exaggerated claim that it can attach corresponding address on every grain of sand and every drop of water of earth. With the rapid development of global internet, 4.3 billion of IP addresses are expected to be in short supply in 2005, of which the shortage is mainly concentrated in Asia and China is the first to be affected.

\section{Design significance}

\subsection{Achieve landing of IPv6 internet}

Explore the physical routing of IPv6 internet accessed in our college, discuss, summarize and complete the connecting scheme of our college, achieve the connection of IPv6 line in our college and achieve related applications based on IPv6 internet, such as WWW、MAIL, DNS and other basic network services, to provide these services for the users in and around our college.

2.2 Establish the IPv6 experimental platform of our college; provide the experimental environment based on IPv6 for the research units in college and provide firsthand information for the education and student cultivation of our college.

At present, NIC of our country has made some research projects combining with network information security institute, of which the various safety tests of IPv6 are essential; and at the same time, with the advance of time and the development of technology, the students of computer and internet specialty in our college shall be indispensably exposed to IPv6 internet, which all will need to lay the foundation of science and technology.

\subsection{Improve information level of college}

Current network of college is based on IPv4 network, on which we can not greatly achieve video conference, Qos, network security and multicast on traditional network. We can carry out various applications on advanced network to improve the level of network operation and management after accessing to IPv6 network. 


\subsection{Provide abundant address resources}

Traditional network is operated on the basis of IPv4 protocol. IPv6 is the next version of the Internet protocol, and the suggestion of it is originally due to that the limited address space of IPv4 shall be used up and the deficiency of address space will affect the further development of network with the rapid development of network.

More than 1,000 addresses can be allocated per square meter of area in the whole global according to the practical assignable address of IPv6 estimated by conservative method. In the process of IPv6 design, the shortage of address shall be solved once and for all, in addition, other unsolvable problems for IPv4 shall be considered.

2.5 Improve the research and application of other relevant new application and new technology in college and build advanced network platform for other departments in college.

2.6 Enter into advanced scientific research, improve the college position, enhance college influence and increase sources of students.

\section{Design principles}

Based on the above analysis for requirements, the design of scheme shall be fully based on international regulations and standards, imitate the system structure of main current network and operation system of network which are popular in domestic and overseas at present and design from the practical requirements of network information. It is ensured that the advancement and practicability of technology make the network with great expandability and flexibility to fit the rapid development of network, which not only meets current requirements but also takes care of the requirements of construction and development of the future network. Make the best of various network resources owned by local to ensure the interconnection of the network inner. Therefore, the regulations for system interoperability shall be insisted while designing network.

3.1 Technical advancement

The plan of network has to combine with the application requirements at present and future, we need to use long-range vision to ensure the science and advancement of plan, and the practicability and advancement of selected equipments, servers and software systems with longer lifecycle and advanced technology level. And at the same time, meet the future business developments combining with practical situation of college.

Presently popular advancement technology is Ethernet technique, experienced developments from the sharing to exchange and from slow to fast. Originally Ethernets are the rate of $10 \mathrm{M}$ and sharing width-type network which is only to make some slow desktop connection; however, present Ethernets not only can get access to working team but also can be used for backbone. 10M/100M Ethernet technology takes hold in working team because that most of the workstations and servers used the Ethernet interface, and gigabit Ethernet technology plays an important role on the basis of simplexes, high speed and great support for upper IP protocol.

\subsection{Network security}

Safety is foundation for network operation, and the network system itself has a high degree of security, providing advanced, reliable, comprehensive security certification program and emergency measures in technology to ensure the system absolutely safe with great hedges for hostile attack from inside and outward of college, which shall adopt various detection and alarm methods for deceit. Be able to nicely bear the main network application which makes it has enough satisfaction of application. Be able to tolerate a certain extent to the impact for large data volume and safety distraction (such as worms, cracker scanning, etc. DOS attack, etc.)

Selected equipments shall provide various safety control system with system-level and flexible to support the user to establish complete safety management system.

3.3 Reliability, usability and maintainability

Network design shall adopt redundancy connection to ensure that the main equipments and lines can be automatic switchover during the failure occurrence. Therefore, the system must have very high reliability from the structure and product performance to ensure the reliability operation of 
system, and try to reduce the influence of network equipment failure, system maintenance and accident on network performance.

The reliability, usability and maintainability of project shall be placed on important place in designing, and prerequisite reliability, usability and maintainability for whole network operation system shall be ensured from the structure design, equipment selection, system construction and network management to ensure that network becomes a constantly operation system.

\section{Implementation plan of IPv6}

\subsection{Mainframe scheme}

In the initial stage of IPv6's development, in the absence of IPv6 research projects and the formation of a wide range of user groups, the mainframe which is only accessed IPv4 protocol and the mainframe which accessed dual stack of IPv4/IPv6 can interconnect with backbone CERNET2 of IPv6 in achieving present IPv4 network under the situation of the formal operation for present IPv4 network infrastructure without stopping and influence.

4.2 Test and analysis of performance

Scheme features: interconnection test between the same IPv4 and IPv6 can be achieved, without difference. From the purpose of building a laboratory, a variety of tests and experiments are required because that the scheme II Pv4/IPv6 dual stack router which used in practical production environment has rarely flexibility, relative to the scheme I. The scheme I has great flexibility which can make various tests and without any influence for present college network.

Suggests for the selection on test scheme: During initial period, scheme I can be selected to carry out various tests and experiments on the basis of practical environment of IPv4/IPv6 interconnection. After the relevant tests and experiments on IPv6, the scheme II shall be suggested to select to improve the present network utter, using the IPv4/IPv6 dual stack router for practical production environment. Gradually the campus network shall be established to the environment supporting IPv6.

\section{Conclusion}

IPv6 has entered into the comprehensive deployment period. To carry out application and scientific research on CERNET2 is an irresistible trend. Accessing to CERNET2, the campus network users can directly visit IPv6 resources, experimental WWW, FTP, DNS, MAIL and other services by tunnel server and meanwhile, because IPv6 is superior to the security feature and infinite address spaces of IPv4, experiments like multicast, streaming media transmission, security isolation and so on can also be tried.

\section{Reference}

[1] Ren W Z, Deng Z L, Lian-Ming X U. Research on Architecture for Digital Campus LBS Based on Grid GIS[J]. Journal of Engineering Graphics, 2010, 31(2):54-58.

[2] Hou S. Implementation of Digital Campus Based on Data Grid[J]. Computer \& Digital Engineering, 2008.

[3] You X, Liu, Shuai. STUDY ON GRID-GIS ARCHITECTURE FRAMEWORK OF DIGITAL CAMPUS BASED ON AGENT[J]. Computer Applications \& Software, 2006.

[4] Cheng H S. The Digital Campus Based on Mobile IPV6[J]. Sci-Tech Information Development \& Economy, 2007.

[5] Chen J, Zou Y, Liu Z, et al. Enabling Grid Computing over IPv6 within a Campus Network[C]// Fourth International Symposium on Parallel Architectures, Algorithms and Programming. IEEE, 2011:285-288. 